\title{
ABZU
}

\section{UNCOVERING THE ORIGIN OF ANCIENT ORGANICS ON MARS}

A mission concept to characterize origin-diagnostic molecular features and patterns in preserved organic matter in ancient Martian muds in situ

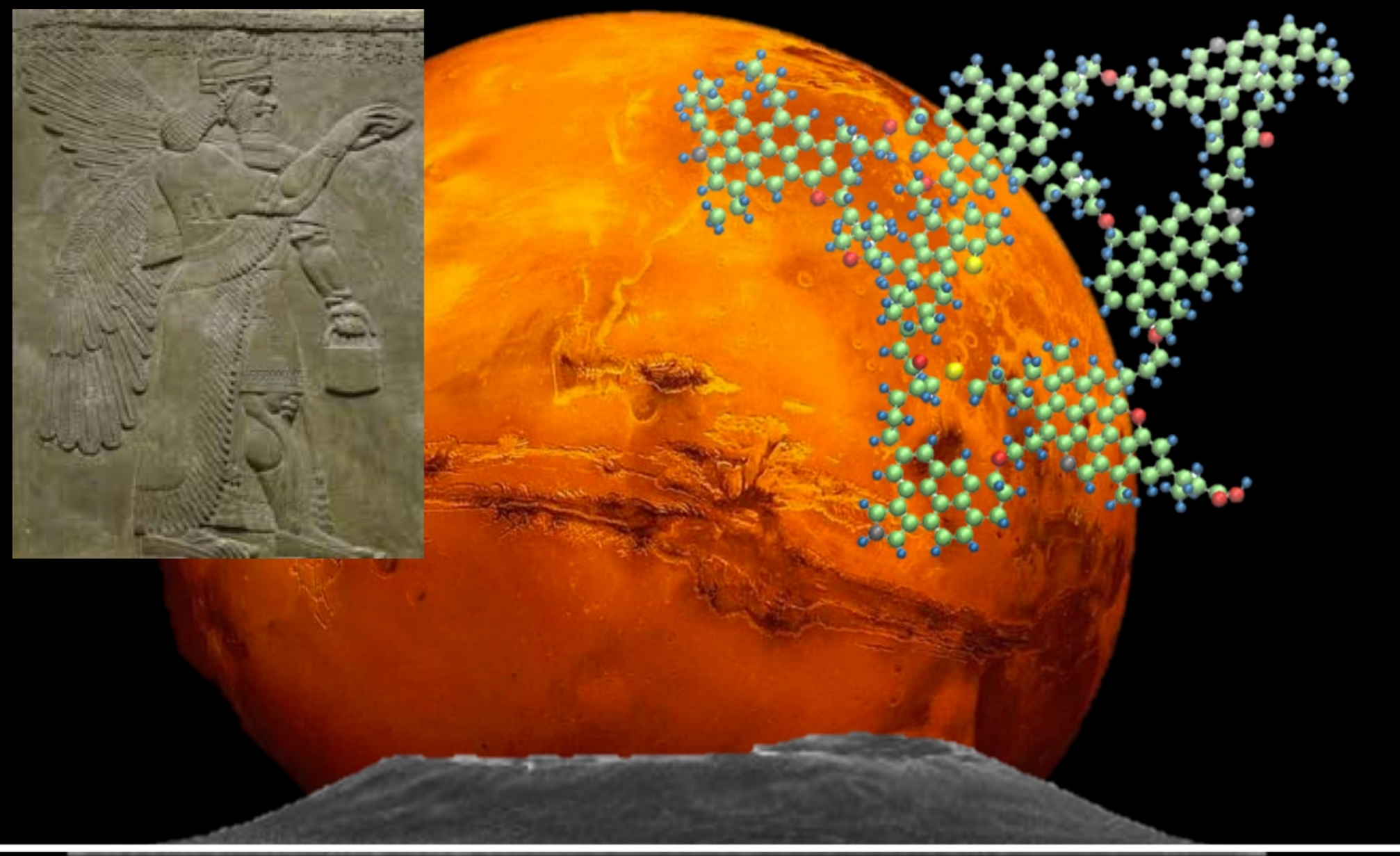

Mary Beth Wilhelm (NASA ARC, marybeth.wilhelm@nasa.gov, (510) 648-0591),

Antonio Ricco (NASA ARC), Dorothy Oehler (PS), Denise Buckner (BMSIS), Alexis Rodriguez (PSI), Paul Mahaffy (NASA GSFC), Jennifer Eigenbrode (NASA GSFC), Mark Ditzler (NASA ARC), Jennifer G. Blank (NASA ARC | BMSIS),

Michel Nuevo (NASA ARC), Pablo Sobron (Impossible Sensing),

Evan Eshelman (Impossible Sensing), Anuscheh Nawaz (APL/UW),

Adrian Southard (NASA GSFC), Ross H. Williams (UMCP), Goro Komatsu (IRSPS), P. Michael Furlong (NASA ARC | SGT), Trey Smith (NASA ARC), Terry Fong (NASA ARC), Jessica Koehne (NASA ARC), Linda Jahnke (NASA ARC) Dave Des Marais (NASA ARC),

Matthew Chin (NASA ARC), Travis Boone (ME)), Tori Chinn (NASA ARC),

Kanch Sridhar (NASA ARC), Thomas McClure (NASA ARC), Trinh Hoac (ME), Morgan J. Anderson (USRA), Leslie Radosevich (ME), Abraham Rademacher (ME), Lauren Friend (NASA ARC), Spencer Baird (Georgia Tech), Thomas Evans (MIT), Jay Bookbinder (NASA ARC), Michael Bicay (NASA ARC) 


\section{Ancient Mars is a key target for life-detection missions.}

Identifying evidence of ancient Martian life would revolutionize our understanding of terrestrial biological processes and predictions for the likelihood of life elsewhere in the Universe. Of all Solar System bodies, Mars must remain a primary life-detection target because conditions during its first 500 million years resembled those on Earth when early life emerged and evolved (e.g. Pollack et al., 1987). One of the best indicators of ancient life on Mars may be preserved organic matter. However, surface degradation of organic matter is a significant impediment to life detection on Mars, as habitable conditions on the surface disappeared billions of years ago (e.g. Ehlmann et al., 2016), the Martian surface receives a high flux of organic-degrading radiation, and unlike Earth, Martian sedimentary facies have not undergone extensive tectonic reworking. To overcome this challenge, Martian life detection strategies must leverage lessons learned from terrestrial biogeochemical investigations: on Earth, billion-year-old molecules are buried in sedimentary rock, preserved in the ancient geological record. Some of these molecules retain biogenic patterns and structures that can elucidate the origin and evolution of life (Summons et al., 2007).

The New Frontiers-sized Abzu mission concept improves on current Mars life-detection strategy by (1) building on recent results of organics on Mars, (2) improving limits of detection (LODs) to relevant levels by leveraging organic biogeochemical extraction and analysis methods routinely applied to terrestrial samples, and (3) targeting a site with accessible ancient Martian sedimentary deposits that offer the best odds of preserved molecular biosignatures. Abzu is named for the groundwater that gave rise to life in ancient Sumerian mythology.

II. Mars life-detection missions must target the accessible, origin-diagnostic organic matter most likely to have survived over geological timescales.

Abzu's mission goal is to determine the origin of organic molecules - biogenic or abiogenic - preserved within ancient Martian sedimentary deposits by characterizing organic matter with the highest preservation potential: lipids and insoluble macromolecular organic material (IMOM). These organic molecules and complexes can survive for billions of years (Fig. 1) and comprise the majority of organic matter preserved in terrestrial rocks, retaining features indicative of their origin (e.g. Georgiou and Deamer 2014).

Organics Detected Previously on Mars: Current understanding of the Martian organic inventory comes from studies of Martian meteorites (Steele et al., 2012; Lin et al., 2014; Steele et al., 2016; Jaramillo et al., 2019) and in situ measurements of the 1976 Viking landers and 2012 Mars Science Laboratory (MSL) Curiosity rover. MSL's Sample Analysis at Mars (SAM) instrument suite discovered chlorinated hydrocarbons (Freissinet et al., 2015) and hydrocarbons likely derived from macromolecular organic matter in ancient lake sediments of Gale crater (Eigenbrode et al., 2018; Freissinet et al., 2019). These findings highlight the potential for hydrocarbon preservation

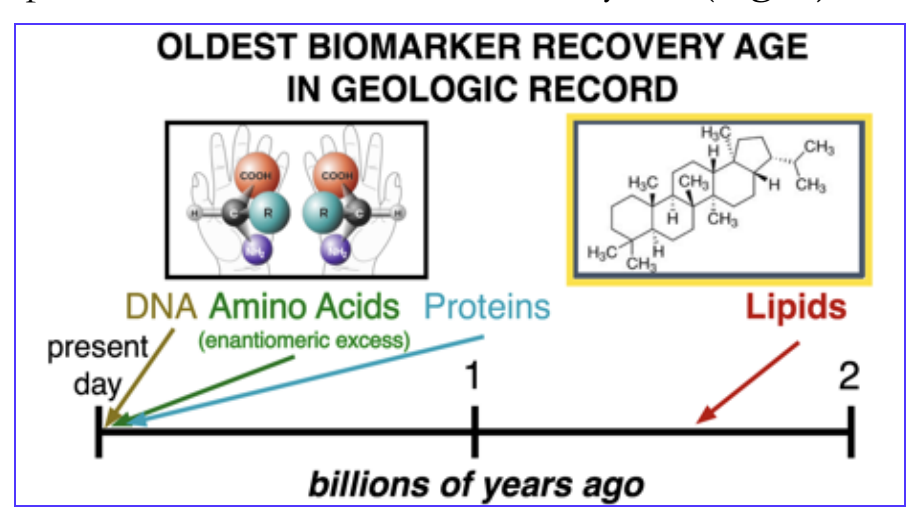

Figure 1. Depiction of oldest biomarker recovery age in the geologic record. Lipids have the greatest longevity in the terrestrial geologic record of any molecular biosignature, outlasting DNA, amino acid enantiomeric excess, and proteins by an order of magnitude. The timescale of their preservation is comparable to the age of the ancient, putatively habitable Martian surface. 
over several billion years on Mars, even after $10^{5}-10^{6}$ years of exposure to ionizing radiation (e.g., Benner et al., 2000; Fox et al., 2019). If life did not arise and become widespread on Mars, lipids could still be present within geologic substrates, perhaps delivered as extraplanetary organic material by meteorites and interplanetary dust particles (see summary in Mahaffy et al., 2012) and endogenous compounds generated in situ through, for example, the electrochemical reduction of atmospheric $\mathrm{CO}_{2}$ (Steele et al., 2018) and/or hydrothermal activity (McCollom et al., 1999). Analyzing origin-diagnostic molecular patterns and features (e.g. Table 1) of Martian samples will help elucidate their astrobiological history.

Proposed Molecular Target \#1 -

Lipids: Lipid biomarkers are of great interest for life- detection missions

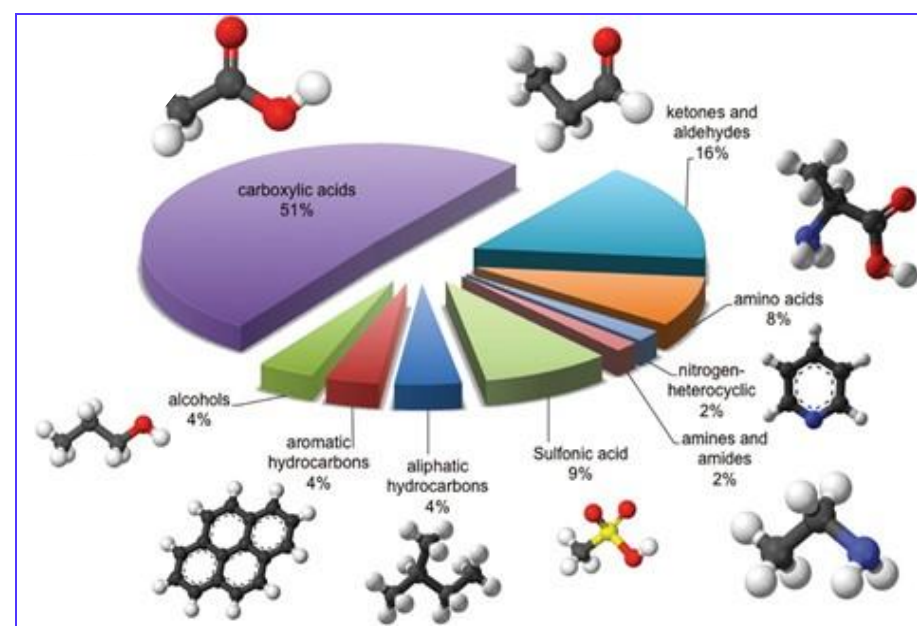

Figure 2. From Remusat (2014) showing that the lipid groups carboxylic acids, aromatic and aliphatic hydrocarbons comprise almost $60 \%$ of soluble organics in Murchison Meteorite (a "type" example of a carbonaceous chondrite). Meteoritic lipids are likely a major source of organic matter deposited on early Earth and Mars during the Late Heavy Bombardment period.

(Summons et al., 2011; Europa Science Definition Team Report, 2016). Lipids are a diverse class of organic molecules that are broadly defined by their solubility in non-polar solvents (Ratnayake and Galli, 2009). On Earth, lipids are ubiquitous and essential to all life, primarily because they make up the cell membranes of all known organisms (e.g. Georgiou and Deamer, 2014). While most lipids on Earth are synthesized biotically, they are also formed abiotically and are the most abundant organics found in carbonaceous meteorites (Fig. 2), They are likely a major source of prebiotic organics on both Earth and Mars (e.g., Sephton, 2005; Aponte et al., 2011; Remusat, 2014).

Preserved lipids (typically hydrocarbon skeletons) are geologically robust. They have high preservation potential and can persist in the terrestrial geologic record for billions of years, an order of magnitude longer than any other biomolecule (Fig. 1) (e.g. Summons et al., 2011). Due to their longevity, preserved lipids may be one of the best indicators of past life in the search for evidence of life on Mars. Extremely arid conditions, like those on Mars over the last $\sim 3.7$ billion years, are expected to enhance structural preservation (Wilhelm et al., 2017), especially in the absence of significant geothermal heating associated with burial, which is largely responsible for postdeposition alteration of organics on Earth. Although Mars' surface receives more radiation than Earth's, burial within regolith can shield organics, slowing radiolytic degradation over geologic timescales. Estimates vary on the burial depths required for effective radiation shielding, but research suggests that $\sim 10 \mathrm{~cm}$ to $\sim 2$ meters of overlying material can shield organics for geologically significant timescales (Osman et al., 2008; Moores and Schuerger, 2012; Pavlov et al, 2012; Eigenbrode et al., 2015; Fox et al., 2019).

Proposed Molecular Target \#2-Insoluble Macromolecular Organic Matter (IMOM): The majority of terrestrial (Vandenbroucke and Largeau, 2007) and extraterrestrial (Septhon, 2002; Pizzarello et al., 2006; Huang et al., 2007; Schmitt-Kopplin et al., 2010; Alexander et al., 2017) organics are bound in IMOM. IMOM is structurally complex, composed primarily of carbon and hydrogen, with lesser amounts of oxygen, nitrogen, sulfur, other light elements, and metals. The high abundance of carbon and long-term preservation of IMOM makes characterization essential to understanding the organic chemistry of a planetary environment. Some lipids containing origin-diagnostic features are 
bound in IMOM (Lee et al., 2019), but require but require chemolytic and/or pyrolytic techniques in order to create analyzable fragments.

Diagnostic Molecular Patterns: Abzu goes beyond organic detection: diagnostic patterns and features in molecular classes and structures will distinguish extracted lipids to determine biotic or abiotic origin. Chromatographic mass spectrometry analyses following sample preparation and biomarker extraction enable characterization of origin-diagnostic features (e.g. Mißbach et al., 2018), including: (1) molecular weight / chain length, (2) chain features (e.g., branching points, double bonds), and (3) isomerization in multiple lipid classes. For example, these parameters can be measured in fatty acids, a lipid class abundant-but different in its details-in both biotic and abiotic systems (Table 1). Deviation from abiotic lipid patterns could indicate biotic origin (e.g. Lovelock, 1965).

Table 1. Comparison of Abiotic and Biotic Patterns in Fatty Acids, a Major Class of Lipids.

\begin{tabular}{|c|c|c|}
\hline $\begin{array}{l}\text { Origin-Diagnostic } \\
\text { Pattern/Feature }\end{array}$ & BIOTIC: Terrestrial Fatty Acids & ABIOTIC: Fatty Acids found in Meteorites \\
\hline $\begin{array}{l}\text { Carbon Chain Length } \\
\text { Maxima and } \\
\text { Distribution }\end{array}$ & $\begin{array}{l}\text { Maxima at chain lengths }>12 \text { carbons, typically } \\
\mathrm{C}_{16} \text { or } \mathrm{C}_{18 .} \\
\text { Unimodal chain length distribution from } \mathrm{C}_{10} \text { to } \mathrm{C}_{24 .} .\end{array}$ & $\begin{array}{l}\text { Maxima at chain lengths of } 1 \text { or } 2 \text { carbons. } \\
\text { Poisson chain length distribution } \\
\text { from } C_{2} \text { to } C_{12}\end{array}$ \\
\hline $\begin{array}{l}\text { Carbon Chain Length } \\
\text { Abundance }\end{array}$ & $\begin{array}{l}\text { Even chain lengths typically more abundant than odd. } \\
\text { Less often, odd chain lengths more abundant. }\end{array}$ & No significant odd/even abundance differences \\
\hline $\begin{array}{l}\text { Branching features in } \\
\text { Hydrocarbon Chain }\end{array}$ & $\begin{array}{l}\text { Isomeric predominance of fatty acids with methyl } \\
\text { branches at iso or anteiso position }\end{array}$ & $\begin{array}{l}\text { High isomeric diversity, ma } \\
\text { species; no preference for }\end{array}$ \\
\hline $\begin{array}{l}\text { Unsaturations in the } \\
\text { Hydrocarbon Chain }\end{array}$ & $\begin{array}{l}\text { Between } 1-6 \text { unsaturations typically present. } \\
\text { Most abundant unsaturated chain lengths } C_{16} \text { or } C_{18} \text {. }\end{array}$ & $\begin{array}{r}\text { Unsaturations rarely occl } \\
\text { unsaturation in } \mathrm{C}\end{array}$ \\
\hline $\begin{array}{l}\text { Presence of cyclic } \\
\text { moieties }\end{array}$ & $\begin{array}{l}\text { Cyclic moieties sometimes present on chain lengths } \\
\text { of } \mathrm{C}_{15}, \mathrm{C}_{17} \text {, or } \mathrm{C}_{19}\end{array}$ & None reported \\
\hline $\begin{array}{l}\text { Abundance of cis vs. } \\
\text { trans isomers }\end{array}$ & Cis conformer in excess & No preference \\
\hline
\end{tabular}

III. Mars' oldest mudstones that have been recently exposed/exhumed should be the target landing sites to search for preserved organic matter.

Abzu's landing site selection criteria include (1) the identification of extensive, topographically smooth terrains (a landing constraint), (2) accessible Early Mars mudstone exposures, (3) sites that may host concentrated organics, and (4) evidence that these mudstones exhibit geologically fresh surfaces. Abzu will target a site of optimal organic accumulation and preservation in a large impact basin in the northern lowlands, likely submerged beneath deep surface water during the Noachian period, that contains large volumes of buried marine sediments (Oehler and Allen, 2012). This region could be among the longest habitable on Mars: 3.7 billion years ago, when the climate cooled and the planet's surface became cryogenic, subsurface permafrost-sealed aquifers could have supported life where liquid water remained stable at depths $>100$ meters, providing a subsurface habitable zone (Dohm et al., 2001). Radar and morphologic data indicate thick, buried water-ice deposits and extensive sedimentary extrusions in this region (Holt et al., 2008). Recent studies suggest mound-like extrusions could possibly be sedimentary volcanoes and diapirs; this could provide access to erupted subsurface material. Such erupted materials are likely to have originally contained high quantities of organics (deposited with fine-grained materials often involved in sedimentary diapirism) initially preserved by rapid burial (Oehler \& Allen 2012). Some of these diapiric structures are thought to be recent, which would diminish the degree of radiolytic degradation due to reduced surface exposure time. These features could be an ideal repository of extraterrestrial life on Mars. We are also exploring geologic features containing ancient Noachian mud recently exposed by geological processes (Rodriguez et al., 2020). 
A Case-Study Region: Assuming a long-lived, progressively shrinking ocean, the deepest areas in the lowlands were likely sites with the longest presence of liquid water, the greatest concentration of organics, and preservation of organics due to rapid burial. These areas may have been some of the longest habitable on Mars. Many of these regions in the northern plains have sedimentary extrusions, interpreted as mud volcanoes (Fig. 3) (Oehler \& Etiope, 2017). Mud volcanism is a type of

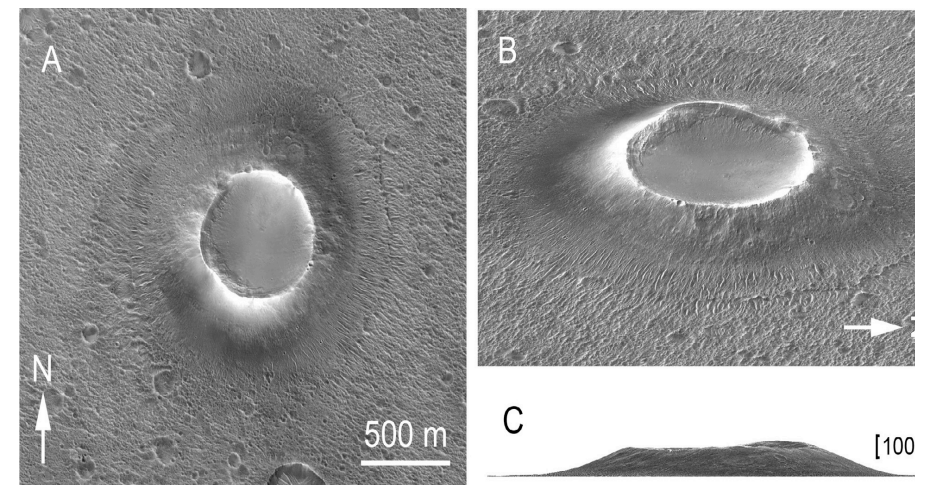

Figure 3. Purported mud volcanoes in southern Chryse Planitia. HiRISE images and 3D perspectives are from HiRISE digital terrain models (from Komatsu et al., 2016). sedimentary volcanism (as opposed to magmatic volcanism) characterized by ascending gaseous sedimentary slurries (typically, water and methane) from unconsolidated sedimentary materials from subsurface sources as deep as several kilometers, deposited early in Mars's history. These deposits are outstanding sites to look for ancient biosignatures (e.g., Komatsu \& Ori, 2000; Oehler \& Allen, 2010; Oehler \& Etiope, 2017). On Earth, mud volcanoes typically involve organic-rich sediments that are transported to the surface with the erupted rocks and boulders.

Clusters of possible mud volcanoes (PMVs) are between $\sim 10^{\circ}$ and $\sim 20^{\circ} \mathrm{N}$ in southern circum-Chryse (Rodriguez et al., 2007; Komatsu et al., 2016; Brož et al., 2019). This region of Mars, which includes the Pathfinder landing site, displays extensive evidence of marine and fluvial sedimentation. Thus far, no lava flows have been identified, which strengthens the hypothesis that the cones are of sedimentary volcanic origin. This region offers multiple potential landing sites for the Abzu mission. The number of cones exceeds 1300, spread over $700,000 \mathrm{~km}^{2}$ (Brož et al., 2019), so we expect numerous landing ellipses would meet the necessary geology characteristics to achieve Abzu's goals. PMVs in southern Chryse Planitia are relatively pristine and crater free and thus appear young (possibly late Amazonian), but their small sizes make precise dating using crater counting difficult. One study constrained the ages of some of the features in that region to as young as 5 million years (based on overlapped secondary craters associated with Mojave crater) (Brož, 2019). The dust cover appears thin ( $\mathrm{mm}$ to $\mathrm{cm}$ ) in most cases, and a PMV morphology is clearly observable. In other cases, minor dune fields cover surfaces of PMVs and such aeolian deposits may reach meters in thicknesses. Because geologically recently exhumed material would have experienced radiation on the surface for relatively short timescales, organics would have undergone less degradation relative to those exhumed by older PMVs: younger PMVs permit shallower sampling to avoid radiolytic degradation of organics/generation of soil oxidants.

\section{Sample selection, processing, and analysis must be based on best-practice techniques for handling of complex, naturally occurring organic matter.}

Front-end sample identification, acquisition, and preparation techniques that conserve origindiagnostic molecular structures/patterns must be used in tandem with analytical methods customized for structural determination of lipids and IMOM. Figure 4 summarizes the overall concept of resultsgated, staged sample collection and analysis. Stand-off characterization/identification of organics should be used first. Next, the location and choice of drilling depth are validated by down-hole in-situ analysis. Validated samples are comminuted onboard the rover for further analysis. Of these samples, 
only the high-value ones are prioritized and delivered to the lander for the most detailed analysis, Fig. 4. Many required payload elements have extensive flight heritage: thermal extraction units, aqueous microfluidic systems, and mass spectrometers.

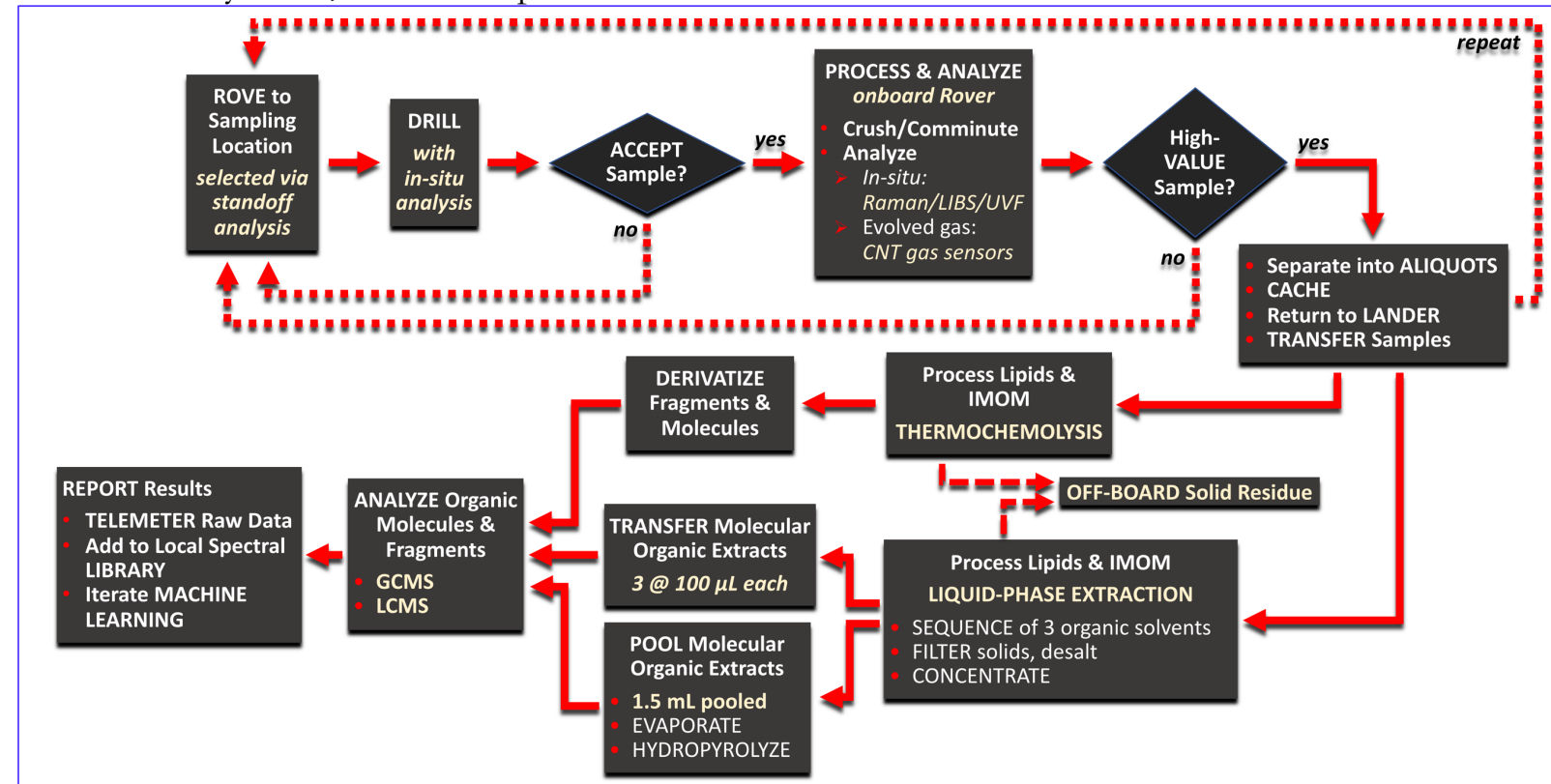

Figure 4. Abzu's notional chain of analyses for life detection in ancient Martian sediments. By performing preliminary analysis of samples, only high-value samples undergo more detailed organic characterization. This approach increases likelihood of organic detection with limited sample numbers.

Organic Content Screening System: In-situ screening of organic content and mineralogy optimizes sample selection, so that resource-intensive detailed analysis is conducted only on samples likely to have sufficient organic content. Raman+LIBS (laser-induced breakdown spectroscopy) is one method to assess sample site mineralogy and geochemistry. Raman spectroscopy provides contextual information about molecular structure and identity-minerals and organic functional groups, including lipids targeted by Abzu with unique Raman spectra (Nakamoto et al., 2009). LIBS provides qualitative and quantitative elemental analysis, as well as mineralogical analysis of the bulk chemical composition; it is sensitive to virtually all elements, especially (i) bio-essential elements including $\mathrm{C}, \mathrm{H}$, N, O, S, P, Fe, and Mn (Creamers et al., 2006); (ii) CN bonds in organic compounds (Baudelet et al., 2007); and (iii) isotopic signatures, including H, B, C, and N isotopic ratios (Doucet et al., 2011; Niki et al., 2008; Russo et al., 2011). A Raman+LIBS system developed through NASA's SBIR/ST'TR program with private investment is currently at Technology Readiness Level (TRL)-5 (Sobron et al., 2019).

Carbon nanotube (CNT)-based gas sensors can also screen samples for relevant organic volatiles. Subsurface samples are heated and volatile emissions detected in a compact instrument as a simplified evolved-gas analysis (EGA), particularly for carbon-bearing molecules such as $\mathrm{CO}, \mathrm{CO}_{2}$, and $\mathrm{CH}_{4}$. High-surface-area $\mathrm{CNT}$ chemiresistors have improved sensitivity and recovery time relative to traditional bulk material counterparts; they can detect trace $\mathrm{NH}_{3}, \mathrm{NO}, \mathrm{SO}_{2}, \mathrm{H}_{2} \mathrm{O}_{2}, \mathrm{CH}_{4}$, and $\mathrm{CO}$ (Li, 2003; Lu, 2011; Popa, 2013; Hannon, 2014, 2016 Meyyappan, 2015). Heritage derives from extensive use of similar gas sensors on the ISS (Stenzel, 2016). These sensors require minimal mass, power, and volume, suiting them to initial sample characterization.

Detailed Analytical Techniques to Reveal Detailed Structural Information: Following organic content screening and organic extraction (see Section $V$ ), detailed analysis will utilize heritage gas chromatograph-mass spectrometry (GC-MS) instrumentation such as the SAM suite (TRL-9), which 
has unit mass resolution from 2-535 Da, dynamic range $\sim 10^{9}$, and an LOD of $\sim 0.5$ ppbw (Mahaffy et al., 2012). Another option is a 50\%-lighter GC-MS ( $20 \mathrm{~kg}$; now TRL-8), part of the MOMA (Mars Organic Molecular Analyzer) instrument on the ExoMars rover, which has comparable performance specifications (Goesmann et al., 2017). By using solvent lipid extraction and with its 50-cc sample size, Abzu will improve upon SAM's LOD by 10 - 3000-fold without loss of origin-diagnostic structural features. Abzu's extraction approach permits more volatile lipids (alkanes, alkenes, aromatic hydrocarbons, smaller acids, alcohols, and ketones) to be volatilized without chemical derivatization; only after such analysis will thermochemolysis and/or derivatization be brought to bear.

Liquid chromatography-mass spectrometry (LC-MS) has a larger analytical window than GCMS and is typically used to target molecules that have a greater range of polarity and size. Liquid-phase extraction using solvents of differing polarity helps reduce the complexity of analytes introduced to the LC, allowing for more organic separation through different solvent extractions. The chemistry of the mobile phase can be tuned to separate species by use of ion-pairing reagents or hydrophobicity (e.g. Parker et al., 2016). LC does not require the analyte to be volatile, lowering the organic alteration risk. To date, system-level implementations of LC-MS are lower TRL ( $\sim 4)$ than those of GC-MS (TRL-9). In one effort (OASIS), an electrospray ionization interface between an LC and miniature time-of-flight mass spectrometer for amino acids was explored by a 2012 ASTID and is at TRL-4. Another effort, MACROS, funded by the PICASSO program, has demonstrated online liquid extraction and deposition into high vacuum with subsequent laser-desorption ionization (LDI) on an amino acid mixture and is at TRL-4. Liquid separations can also be achieved through capillary electrophoresis (CE) for amino acids (Creamer et al. 2017), but this yet to be demonstrated in planetary instrumentation for a wide range of lipids. Finally, tandem MS analytical instrumentation (e.g. Kaplan et al., 2019) should be explored further, as it can interrogate the intrinsic complexity of a molecule, an agnostic biosignature (Marshall et al., 2017).

Sample Analysis Operations and Automation: Multiple levels of screening (Fig. 4) optimize use of the limited reagents and resources of a planetary lander/rover-based mission. Preparations for Abzu will include laboratory and field studies of a wide range of field and analog samples in which target lipid concentrations are manipulated to match an anticipated range of relevant possibilities on Mars. These studies will build a multi-parameter sample-analysis methods database targeted at conserving limited resources: sample volume, reagents, energy, and time. During the mission, these methods will improve verification and validation of results obtained via integrated information from Abzu instruments. We are currently exploring their use to tune instrumentation operating parameters autonomously during the mission.

V. Best-practice sample preparation is crucial for identifying origin-diagnostic molecular information for life detection.

Previous Mars missions discovered trace quantities of organic matter, but origin-diagnostic features (i.e. abiotic vs. biotic) have been obscured by pre-analytical processing (i.e. thermal extraction). Constraints placed on SAM at its selection led to significant descoping of its preanalytical sample-processing capabilities, preventing extraction protocols more advanced than thermochemolysis. MOMA on the upcoming ExoMars mission will continue the search for organics in a clay-rich region (Goessman et al., 2018), implementing chemical derivatization and LDI-MS within its mass and energy usage constraints.

Abzu will employ more advanced organic extraction and analytical techniques to overcome experimental limitations that impacted SAM. Use of best-practice laboratory methods adapted for spaceflight with comparatively large sample quantities for characterization of lipids and 


\begin{abstract}
IMOM addresses common analytical challenges such as low organic abundance, interference minerals/salts, degradation of diagnostic of of originstructures. Critically, this approach can reveal such origin-diagnostic information to distinguish biogenic from abiogenic signatures by mitigating detection ambiguities and maximizing organic signal-tobackground ratios. Abzu will apply this approach to characterization of organic material on Mars for the first time with the Extractor for Chemical Analysis of Lipid Biomarkers in Regolith
\end{abstract} (ExCALiBR) (currently TRL-4,

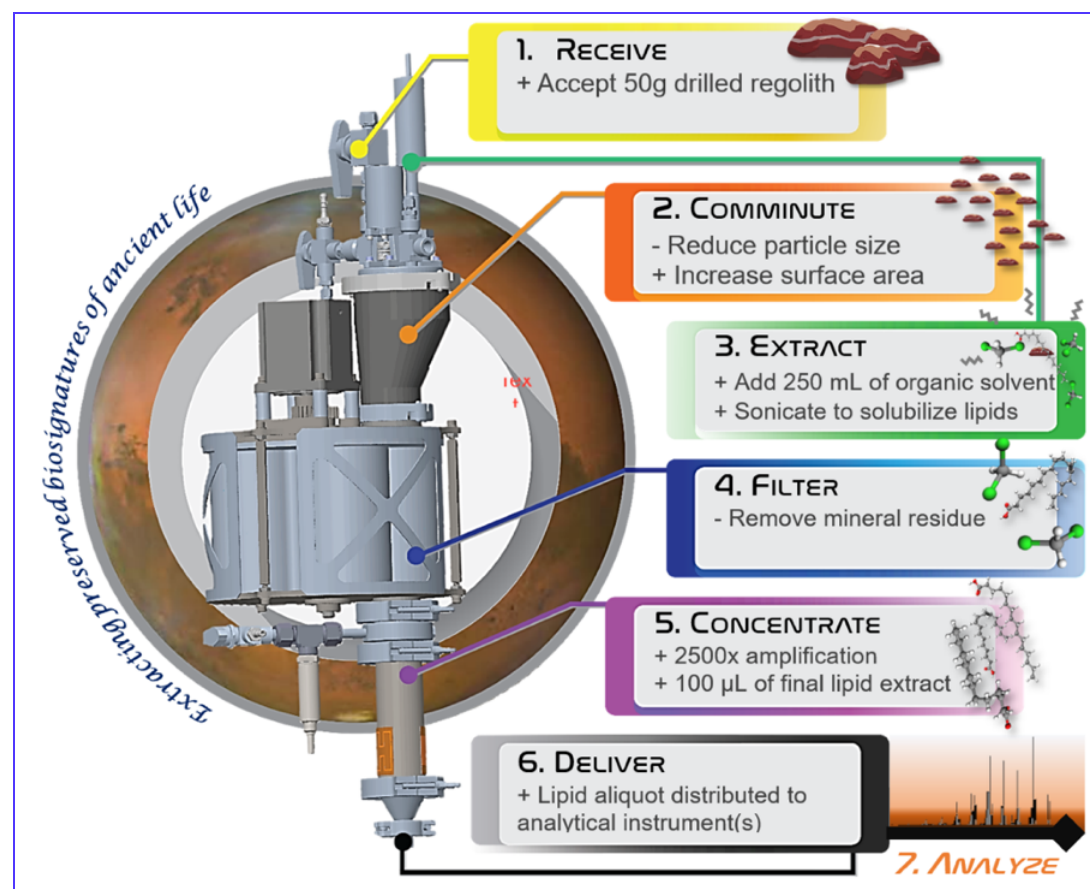

Figure 5: The ExCALiBR system (TRL-4) implements six key steps to extract lipids from rock powders on Mars and deliver the extracts to instruments for analysis. Functions and corresponding enabling subunits are shown.

funded through a NASA STMD Early Career Initiative award) that optimizes solvent extraction and concentration of lipids for delivery to instruments for molecular characterization (Fig. 5). Unlike sample-return missions, sample mass is not significantly restricted, and the capability to handle much larger $(50 \mathrm{cc})$ samples will result in much larger signals and lower LODs from extracted organics. Wellestablished liquid-phase lipid extraction/preparation approaches effectively overcome common challenges in analysis of natural samples (i.e., planetary analog samples) by: (1) disrupting molecular interactions between minerals and organics (Kiel \& Mayer, 2014); (2) increasing concentration to enable detection of low-abundance biomass (Karl et al., 1999; Wilhelm et al., 2017, 2018); (3) avoiding deleterious reactions with oxychlorine anions (Lewis et al., 2015). To date, no mission has replicated optimal laboratory approaches to organic/lipid extraction and characterization. Use of these techniques also enables scientists to leverage 80 years' worth of research detailing the organic content of a vast diversity of sample types. This is tremendously advantageous for analysis and interpretation of samples on Mars with a priori relatively unknown organic content.

REFERENCES: Alexander, C. M. O’. D., et a., (2017). Chemie der Erde-Geochemistry, 77. | Aponte, J. C., et al., (2011). Geochim. et Cos. Acta, 75. | Brož, P., et al., (2019 JGR-Planets, 124. | Dartnell, L. R. et al., (2007). GRL, 34. | Eigenbrode, J. L., et al. (2018). Science, 360. | Eigenbrode, J., et al., (2009). Astrobio, 9. | Fox, A. C., et al., (2019). GR: Planets, 124. | Freissinet, C., et al. (2015). J. Geophys. Res. Planets, 120. | Freissinet, C., et al., (2019). LPICo, 2089, 6123. | Georgiou, C. D., \& Deamer, D. W. (2014). Astrobio, 14. | Goesmann, F., et al. (2017). Astro, 17(6-7), 655-685. | Hannon, A., et al., (2014). Sensor Lett., 12. | Kaplan, D.A., et al., (2019) 13"h HEMS Workshop. | Hannon, A., et al.. (2016). Sensors, 16. | Huang, Y., et al., (2007). EPSL, 259. | Jaramillo, E. A., et al., (2019). GRL, 46. | Karl, D., et al., (1999). Science, 286. | Keil, R. G., \& Mayer, L. M. (2014). Treatise on Geochem 12. | Komatsu, G., \& Ori, G. G. (2000). Planet. and Space Sci, 48. | Komatsu, G., et al., (2016). Icarus, 268. | Lee, C., et al.,(2019). Organic Geochem. | Lewis, J. M., et al., (2015). Astrobio., 15. | Li, J., et al., (2003). Nano Letters, 3. |Lin, Y., et al., (2014). Meteoritics \& Planet. Sci., 49. | Lovelock, J. E. (1965). Nature, 207. | Lu, Y., et al., (2011). Small, 7. | Mahaffy, P. R., et al. (2012). Space Sci. Rev., 170. | Marshall, S. M., et al., (2017). Phil. Trans. of the Royal,375. | Meyyappan, M., et al., (2015). MRS Bulletin, 40. | Mißbach, H., et al., (2018). Organic Geochem, 119. | Moores, J.E., \& Schuerger, A.C. (2012). | Oehler, D. Z., \& Allen, C. C. (2010). Icarus, 208. | Oehler, D. Z., \& Etiope, G. (2017). Astrobio, 17. | Osman, S., et al., (2007). 74. | Parker, E. T., et al., (2016).. Rapid Commun. Mass Spectrom., 30. | Pavlov, A. A., et al., (2012). GRL, 39. | Pizzarello, S., et al., (2006). Meteorites and the early solar system II, 1. | Popa, A., Li, J., \& Samia, A. C. S. (2013). Hybrid platinum nanobox/carbon nanotube composites for ultrasensitive gas sensing. Small, 9, 3928-3933. | Ratnayake, W. N., \& Galli, C. (2009). Annals of nutrition \& metabolism, 55. Remusat, L. (2014). BIO Web of Conferences, 2. | Rodriguez, J. A. P., et al., (2007). Icarus, 191. | Rodriguez, J. A. P., et al., (2020). Mature Scientific reports, 10. | Russo, R.E., et al. (2011). Spectrochim. Acta Part B: Atomic Spectroscopy, 66. | Schmitt-Kopplin, P., et al.,(2010). PNAS, 107. | Sephton, M. (2005). Phil.Trans 363. | Sephton, M. A. (2002). Natural product reports, 19. | Sobron P., et al.,(2018). Electronic New Technology Report (NNA15BB01A). | Steele, A., et al., Sci adv., 4. | Steele, A., et al., Meteor. \& Plan. Sci., 51. | Steele, A., et al., (2012). Sci., 337. | Stenzel, C. (2016). Anal. Bioanal. Chem., 408. | Summons, R. E., et al., (2011). Astrobio,11. | Vandenbroucke, M., \& Largeau, C. (2007). Org. Geochem, 38. | Wilhelm, M.B., et al., (2017). Org. Geochem.,103. | Wilhelm, M.B. et al., (2018). Astrobio,18. 